\title{
Asymptotics of the Far Fields of Internal Gravity Waves Excited by a Source of Radial Symmetry
}

\author{
V. V. Bulatov ${ }^{a, *}$ and Yu. V. Vladimirov ${ }^{a, * *}$ \\ ${ }^{a}$ Ishlinsky Institute for Problems in Mechanics of the Russian Academy of Sciences, Moscow, Russia \\ *e-mail: internalwave@mail.ru \\ **e-mail:vladimyura@yandex.ru
}

Received February 11, 2021; revised March 23, 2021; accepted March 23, 2021

\begin{abstract}
The problem of the far field of internal gravity waves generated by a perturbation source of radial symmetry aroused at an initial instant of time is solved. The constant model distribution of the buoyancy frequency is considered and, using the Fourier-Hankel transform, an analytical solution to the problem is obtained in the form of the sum of wave modes. Asymptotics of the solutions that describe the spatial-temporal characteristics of elevation of the isopycnic lines and the vertical and horizontal velocity components far from the perturbation source are obtained. The asymptotics of the components of the wave field are expressed in terms of the square of the Airy function and its derivatives in the neighborhood of the wave fronts of an individual wave mode. The exact and asymptotic results are compared and it is shown that the asymptotic method makes it possible to calculate effectively the far wave fields at times of the order of ten and more of the Brunt-Väisälä periods.
\end{abstract}

Key words: stratified medium, internal gravity waves, buoyancy frequency, far fields, burst source

DOI: $10.1134 / \mathrm{S} 0015462821050013$

The analytical and numerical investigations of the evolution of dispersive internal gravity waves (IGW) generated by nonlocal perturbation sources in natural stratified media show that the structure of wave patterns at large distances from these sources (much more than their characteristic dimensions) depends only slightly on their shape and is determined by only the dispersion laws of these media [1-3]. Therefore, using model representations to describe various sources, in the far zone the wave field can be described using relatively simple analytical formulas [4-6]. In this case the initial and boundary conditions must be determined from the results of direct numerical simulation of the near field with account for the nonlinear hydrodynamic equations or using particularly evaluating (semi-empirical) considerations [6-8]. The assumption on excitation of IGW packets by the pulse action $[4,5,9-11]$ may be considered as a possible model of wave generation. To carry out estimative calculations of internal gravity waves it is necessary to select the parameters of the used source model so that to approximate the modeled wave systems to those observed in reality including the space photos and wave patterns [12-14]. Thus, the mathematical models of wave generation can be not only verified but also used to carry out the predictive estimations since these model conditions specified a priori contain a lot of real information on whose base the linear theory can give satisfactory results far from various perturbation sources [3, 4, 15-18].

The aim of the present study is to construct asymptotics that describe the far fields of linear internal gravity waves excited by a perturbation source of radial symmetry aroused at an initial instant of time in the layer of a stratified medium of finite thickness.

\section{FORMULATION OF THE PROBLEM}

We will consider the layer of a stratified medium of finite thickness $H$. In the cylindrical coordinates $(r, z)$ (it is assumed that the quantities are independent of the angle and the $z$ axis is directed upward) in the Boussinesq approximation the equation for linear internal gravity waves for small perturbations of the isopycnic elevation $\eta(r, z, t)$ takes the form $[1,6]$ :

$$
\frac{\partial^{2}}{\partial t^{2}}\left(\Delta+\frac{\partial^{2}}{\partial z^{2}}\right) \eta(r, z, t)+N^{2}(z) \Delta \eta(r, z, t)=0
$$




$$
\Delta=\frac{\partial^{2}}{\partial r^{2}}+\frac{1}{r} \frac{\partial}{\partial r}
$$

Here and in what follows the Brunt-Väisälä frequency (the buoyancy frequency) is assumed to be constant $N^{2}(z)=N^{2}=$ const. The initial and boundary conditions are taken in the form:

$$
\begin{gathered}
\eta(r, z, 0)=\eta_{0}(r, z)=\Phi(r) \Pi(z), \\
\frac{\partial \eta(r, z, t)}{\partial t}=W(r, z, t)=0 \quad \text { at } \quad t=0, \\
\eta(r, z, t)=0 \quad \text { at } \quad z=0,-H,
\end{gathered}
$$

where $W(r, z, t)$ is the vertical velocity component and the initial perturbation of the isopycnic lines $\eta_{0}(r, z)$ is assumed to have radial symmetry. All the unknown functions depend on the radial coordinate $r$, time $t$, and the vertical coordinate $z$, while there is no dependence on the angle. The solution of the initialboundary-value problem obtained will be constructed using the Fourier-Hankel transform [19, 20]. As a result, we obtain

$$
\begin{gathered}
\eta(r, z, t)=\sum_{n=1}^{\infty} \eta_{n}=\sum_{n=1}^{\infty} a_{n} \varphi_{n}(z) g_{n}(r, t), \quad \varphi_{n}(z)_{n}=\sin (\pi n z / H), \quad a_{n}=\frac{2}{H} \int_{-H}^{0} \Pi(z) \varphi_{n}(z) d z, \\
g_{n}(r, t)=\int_{0}^{\infty} A(k) k J_{0}(k r) \cos \left(\omega_{n}(k) t\right) d k, \quad \omega_{n}(k)=k N / \sqrt{k^{2}+(\pi n / H)^{2}}, \quad A(k)=\int_{0}^{\infty} r J_{0}(k r) \Phi(r) d r,
\end{gathered}
$$

where $J_{0}$ is the zero-order Bessel function. Note that the function $A(k)$ is independent of the mode number $n$ by virtue of constant buoyancy frequency and $g_{n}(r, 0)=\Phi(r)$ for all the numbers $n$. The expressions for the vertical velocity component take the form:

$$
W(r, z, t)=\sum_{n} W_{n}=\sum_{n} a_{n} \varphi_{n}(z) p_{n}(r, t), \quad p_{n}(r, t)=-\int_{0}^{\infty} A(k) J_{0}(k r) \omega_{n}(k) \sin \left(\omega_{n}(k) t\right) d k .
$$

The horizontal (radial) velocity component $U(r, z, t)$ can be determined from the incompressibility equation in the cylindrical coordinates [6]

$$
\frac{\partial U}{\partial r}+\frac{U}{r}+\frac{\partial W}{\partial z}=0
$$

Taking into account the fact that the solution of the equation $[19,20]$

$$
\frac{\partial Y(k r)}{\partial r}+\frac{Y(k r)}{r}=J_{o}(k r)
$$

is the function $Y(k r)=J_{1}(k r) / k\left(J_{1}\right.$ is the first-order Bessel function), we can obtain

$$
\begin{gathered}
U(r, z, t)=\sum_{n=1}^{\infty} U_{n}=\sum_{n=1}^{\infty} d_{n} \psi_{n}(z) q_{n}(r, t), \\
\psi_{n}(z)=\cos \left(b_{n} z\right), \quad d_{n}=\pi n a_{n} / H, \quad q_{n}(r, t)=\int_{0}^{\infty} A(k) J_{1}(k r) \omega_{n}(k) \sin \left(\omega_{n}(k) t\right) d k .
\end{gathered}
$$

The obtained solutions (1.3) show that the horizontal (radial) velocity component $U(r, z, t)$ is equal to zero at $r=0$ and all the values of $z$, $t$, i.e., $U(0, z, t) \equiv 0$. In dimensionless variables $r^{*}=r \pi / H, z^{*}=z \pi / H$, $k^{*}=k \pi / H$, and $\tau=N t$ (in what follows, the symbol "*" will be omitted) the expressions (1.1) $-(1.3)$ can be represented in the form:

$$
g_{n}(r, \tau)=\int_{0}^{\infty} A(k) k J_{0}(k r) \cos \left(\omega_{n}(k) \tau\right) d k
$$




$$
\begin{gathered}
p_{n}(r, \tau)=-\int_{0}^{\infty} A(k) k J_{0}(k r) \omega_{n}(k) \sin \left(\omega_{n}(k) \tau\right) d k, \\
q_{n}(r, \tau)=\int_{0}^{\infty} A(k) J_{1}(k r) \omega_{n}(k) \sin \left(\omega_{n}(k) \tau\right) d k, \\
\varphi_{n}(z)=\sin (n z), \quad \psi_{n}(z)=\cos (n z), \quad a_{n}=\frac{2}{\pi} \int_{-\pi}^{0} \Pi(z) \sin (n z) d z, \quad d_{n}=n a_{n}, \quad \omega_{n}(k)=k / \sqrt{k^{2}+n^{2}} .
\end{gathered}
$$

\section{ASYMPTOTICS OF THE SOLUTIONS IN THE NEIGHBORHOOD OF WAVE FRONTS}

In the given initial distribution of elevation of the isopycnic lines $\eta_{0}(r, z)$ we will assumed that the functions $\Phi(r)$ and $\Pi(z)$ are normalized by their maxima (taken in absolute value). Then, we will consider the following initial radial distribution of the initial perturbation: $\Phi(r)=\exp \left(-r^{2} / 4\right) / 2$ (the factor $1 / 2$ is used for simplicity of algebra). Then from (1.1) we will have: $A(k)=\exp \left(-k^{2}\right)$. For large values $r \gg 1$ and $\tau \gg 1$ the integrals (1.4) can be calculated using the steady-state phase method. For this purpose it is necessary to replace the Bessel function by its asymptotics $J_{0}(k r) \approx \sqrt{2 / \pi k r} \cos (k r-\pi / 4)[19,20]$. Substituting this expression in (1.4), we can obtain

$$
\begin{gathered}
g_{n}(r, \tau)=\sqrt{2 / \pi r} \int_{0}^{\infty} \exp \left(-k^{2}\right) \sqrt{k} \cos (k r-\pi / 4) \cos \left(\omega_{n}(k) \tau\right) d k=I_{n}^{+}+I_{n}^{-}, \\
\left.I_{n}^{+}=\sqrt{1 / 2 \pi r}\right) \int_{0}^{\infty} \exp \left(-k^{2}\right) \sqrt{k} \cos \left(k r \pm \omega_{n}(k) \tau-\pi / 4\right) d k
\end{gathered}
$$

For large values of $r$ and $\tau$ the integral $I_{n}^{+}$is exponentially small since there are no stationary points on the integration interval. Using the steady-state phase method, we can obtain the following equation for finding the stationary points: $\omega_{n}^{\prime}(k)=\rho$, where $\rho=r / \tau$. The solution of this equation takes the form: $k_{n}(\rho)=$ $n \sqrt{(\rho n)^{-2 / 3}-1}$. Finally, we can obtain: $g_{n}(r, \tau) \approx G_{n}(r, \tau) \cos \left(\Phi_{n}(r, \tau)\right)$, where $\Phi_{n}(r, \tau)=\tau\left(1-(n \rho)^{2 / 3}\right)^{3 / 2}$. Similarly, using the steady-state phase method, we have $p_{n}(r, \tau) \approx P_{n}(r, \tau) \sin \left(\Phi_{n}(r, \tau)\right), P_{n}(r, \tau)=$ $-\left(1-(n \rho)^{2 / 3}\right)^{1 / 2} G_{n}(r, \tau)$, and $q_{n}(r, \tau) \approx Q_{n}(r, \tau) \cos \left(\Phi_{n}(r, \tau)\right)$. The obtained asymptotic formulas for the functions $g_{n}(r, \tau), p_{n}(r, \tau)$, and $q_{n}(r, \tau)$ make it possible to calculate the spatial and temporal characteristics of elevations of isopycnic lines and the vertical and horizontal (radial) velocity components of internal gravity waves in the approximation of the steady-state phase at a fixed depth far from a nonlocal perturbation source of radial symmetry which is burst at the initial instant of time. However, these asymptotics cannot be used in the neighborhood of wave fronts [6,20]. To construct the local asymptotics, i.e., the asymptotics that describe the field of internal gravity waves, in what follows we will replace the function $\omega_{n}(k)$ in the integral $I_{n}^{-}$for small wavenumbers by the expansion $\omega_{n}(k) \approx k / n-k^{3} n^{-3} / 2$. Then we can obtain

$$
g_{n}(r, \tau) \approx \sqrt{/ 2 \pi r} \int_{0}^{\infty} \exp \left(-k^{2}\right) \sqrt{k} \cos \left(k^{3} r n^{-3} / 2-\xi k-\pi / 4\right) \cos \left(\omega_{n}(k) \tau\right) d k, \quad \xi=\tau / n-r .
$$

For small $\xi$ (in the neighborhood of wave fronts) stationary points tend to zero, i.e., to the edge of the integration domain and simultaneously to the singularity of the integrand $\sqrt{k}$. In this case the steady-state phase method cannot be used and to construct local asymptotics the original integral has to be reduced to a more complex standard integral using a suitable change of variables. The choice of the standard integral is determined by the distribution of the stationary points of the phase function and the singular points of the integrand as a function of the problem parameters. Construction of local asymptotics is based on reduction of the original integral to a standard integral, i.e., such a simplest integral which has the required set of critical points located similarly to their mutual location in the integral under consideration. Thus, the construction of asymptotics reduces to choice of the corresponding special function and its several 
first derivatives, and to determination of the dependence of arguments of this special function, as well as the amplitude and phase multiplier as functions of the problem parameters. In this case the model integral which can be expressed in terms of the square of the Airy function will be the following integral [20, 21]:

$$
\begin{gathered}
G(x)=\int_{0}^{\infty} \frac{1}{\sqrt{u}} \cos \left(u^{3} / 12-x u-\pi / 4\right) d u=2 \pi^{3 / 2} A i^{2}(x), \\
A i(x)=\frac{1}{2 \pi} \int_{0}^{\infty} \cos \left(u^{3} / 3-x u\right) d u .
\end{gathered}
$$

The function $G(x)$ must satisfy the equation $G^{\prime \prime \prime}(x)=4 x G^{\prime}(x)+2 G(x)$ that can be solved by the Laplace method [20,21]. Then, using the change of variable $k=\operatorname{tn}(6 \tau)^{-1.3}$, we can obtain the following expressions for local asymptotics in the neighborhood of the wave front that take the form:

$$
\begin{gathered}
g_{n}(r, \tau) \approx \frac{n^{3 / 2} J(\Theta)}{2 \sqrt{3 \pi r \tau}}, \quad \Theta=(\tau-n r)(6 \tau)^{-1.3}, \\
J(x)=G^{\prime}(x)=4 \pi^{3 / 2} A i(x) A i^{\prime}(x)=\int_{0}^{\infty} \sqrt{u} \cos \left(u^{3} / 12-x u-\pi / 4\right) d u .
\end{gathered}
$$

For the functions $p_{n}(r, \tau)$ the local asymptotics in the neighborhood of the wave fronts can be obtained from (2.1) by means of differentiation with respect to the variable $\tau$ (in this case only the function $J$ should be differentiated)

$$
p_{n}(r, \tau) \approx \frac{n^{3 / 2} F(\Theta)}{(6 \tau)^{5 / 6} \sqrt{2 \pi r}}, \quad F(\Theta)=4 \pi^{3 / 2}\left(A i^{\prime}(\Theta)^{2}-\Theta A i(\Theta)^{2}\right) .
$$

The expressions for the local asymptotics of the horizontal (radial) velocity component take the form:

$$
q_{n}(r, \tau) \approx \frac{n^{1 / 2} J(\Theta)}{2 \sqrt{3 \pi r \tau}}
$$

We can note that the expressions for the asymptotics of elevation of the isopycnic lines $g_{n}(r, \tau)$ and the horizontal (radial) velocity component $q_{n}(r, \tau)$ coincide correct to the factor $n$ (number of mode).

\section{RESULTS OF THE NUMERICAL CALCULATIONS}

For the numerical calculations we used the following representation of the function $\Pi(z)$ that has a single maximum, namely, $\Pi(z)=z^{\alpha}\left(1-z^{\beta}\right)$, the values of the parameters were as follows: $\alpha=33$, $\beta=57$. The spatial scales used and the character of variability of the initial perturbation of the isopycnic lines correspond to the typical horizontal and vertical scales of real sources of excitation of internal gravity waves in the Ocean [3-5, 12-14]. In Fig. 1 we have reproduced the results of calculations of the function $g_{1}(r, \tau)$ (the first elevation mode) at $\tau=30$ and $\tau=70$ (the left- and right-hand pictures, respectively). Continuous curve corresponds to the exact solution and broken curve to the approximation of local asymptotics on the base of formula (2.1). From the represented results we can see good coincidence of the exact and asymptotic results in the neighborhood of the wave fronts for large $r, \tau$. In Fig. 2 we have reproduced the results of calculations of the function $g_{1}(r, \tau)$ (the first elevation mode) at $\tau=70$. Continuous curve corresponds to the exact solution, dashed curve to the calculations based on the steady-state phase method, and dotted curve to the approximation of local asymptotics from formula (2.1). As shown by the numerical calculations, the asymptotics obtained make it possible to calculate fairly exactly the far wave fields at times of the order of ten and more of the Brunt-Väisälä periods. In the neighborhood of the wave fronts of an individual mode the asymptotics of components of the wave field of internal gravity waves (elevation of the isopycnic lines and the vertical and horizontal velocity components) can be expressed in terms of square of the Airy function and its derivatives, the steady-state phase method can be used at large distances from the wave fronts.

The general scheme of modeling the far fields of internal gravity waves generated by a suddenly appearing nonlocal perturbation source can be represented as follows. Using the numerical solution of the complete system of hydrodynamic equations, the basic characteristics of parameters of the wave field (the iso- 

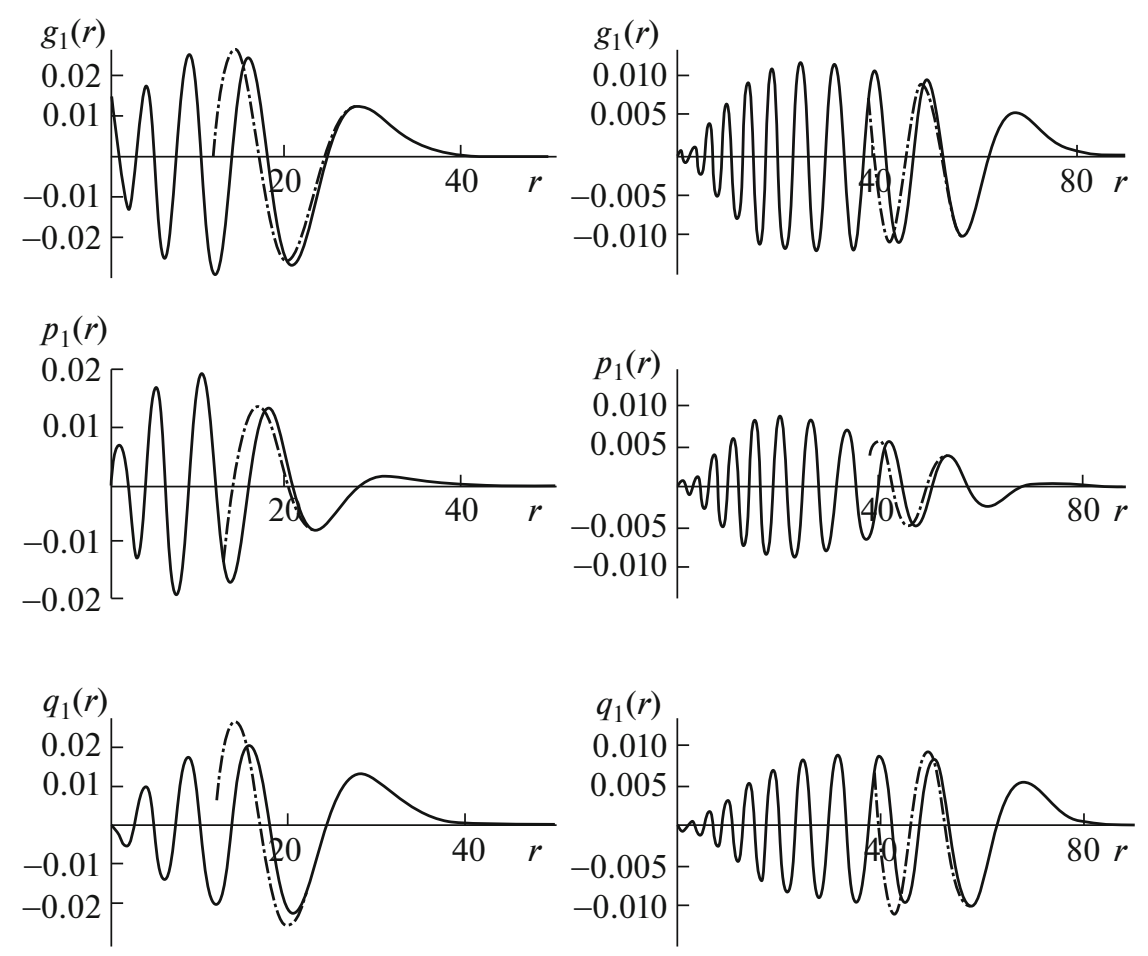

Fig. 1. Elevation of the first mode: exact solution and local asymptotics on the neighborhood of the front.

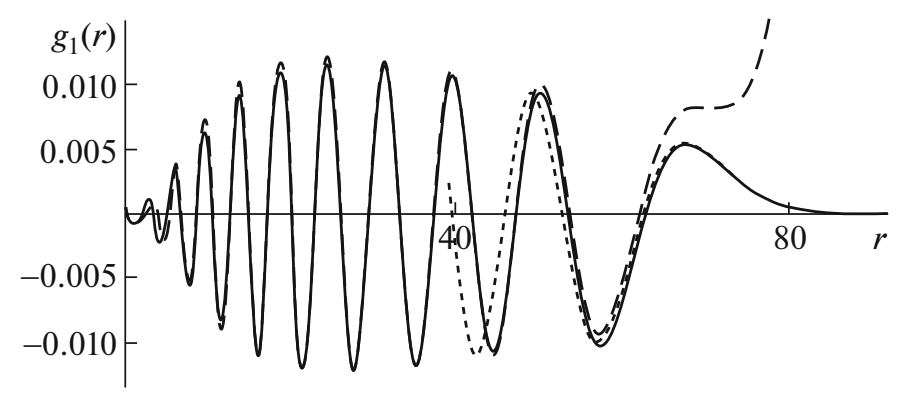

Fig. 2. Elevation of the first mode: exact solution, local asymptotics, and asymptotics of steady-state phase.

pycnic elevation, the velocity components, the density, and the pressure) can be determined; a certain initial spatial distribution of these components can be specified [6-8]. Far from the perturbation sources, assuming the adequacy of using the linear wave dynamics model, the far fields of internal gravity waves can be calculated from the asymptotic formulas; in this case, as shown by the calculation results, under the majority of real hydrological conditions of the World Ocean the basic contribution to the far fields is made only by first several wave modes [3, 4, 16-18].

\section{SUMMARY}

The asymptotics of solutions obtained in the present study make it possible effectively to calculate the wave fields of internal gravity waves far from the nonlocal perturbation sources and qualitatively estimate the solutions obtained. As a result of carrying out multivariant model calculations on the base of the asymptotic formulas, the modeled wave system can be approached to the wave systems observed under the natural conditions. This makes it possible to estimate the physical parameters of real sources of excitation of internal gravity waves in the ocean. Therefore, the asymptotic results obtained make it possible to determine the basic characteristics of the initial perturbations by varying the model values of the initial parameters. 


\section{FUNDING}

The work was carried out with financial support from the Russian Foundation for Basic Research (project no. 20-01-00111A).

\section{OPEN ACCESS}

This article is licensed under a Creative Commons Attribution 4.0 International License, which permits use, sharing, adaptation, distribution and reproduction in any medium or format, as long as you give appropriate credit to the original author(s) and the source, provide a link to the Creative Commons license, and indicate if changes were made. The images or other third party material in this article are included in the article's Creative Commons license, unless indicated otherwise in a credit line to the material. If material is not included in the article's Creative Commons license and your intended use is not permitted by statutory regulation or exceeds the permitted use, you will need to obtain permission directly from the copyright holder. To view a copy of this license, visit http://creativecommons.org/licenses/by/4.0/.

\section{REFERENCES}

1. Lighthill, J., Waves in Fluids, Cambridge: Cambridge University Press, 1978.

2. Konyaev, K.V. and Sabinin, K.D., Volny vnutri okeana (Waves inside Ocean), Saint-Petersburg: Gidtometeoizdat, 1992.

3. Morozov, E.G., Oceanic Internal Tides. Observations, Analysis and modeling. Berlin: Springer, 2018.

4. The Ocean in Motion, Ed by Velarde, M.G., Tarakanov, R.Yu., and Marchenko, A.V., Springer Oceanography. Springer International Publishing AG, 2018.

5. Mei, C.C., Stiassnie, M., and Yue, D.K.-P., Theory and Applications of Ocean Surface Waves, London: World Scientific Publishing, 2017.

6. Bulatov, V.V. and Vladimirov, Yu.V., Volny v stratifitsirovannykh sredakh (Waves in Stratified Media), Moscow: Nauka, 2015

7. Gushchin, V.A. and Matyushin, P.V., Simulation and study of stratified flows around finite bodies, Computational Mathematics and Mathematical Physics, 2016, vol. 56, no. 6, pp. 1034-1047.

8. Matyushin, P.V., Process of the formation of internal waves initiated by the start of motion of a body in a stratified viscous fluid, Fluid Dynamics, 2019, vol. 54, no. 3, pp. 374-388. https://doi.org/10.1134/S0015462819020095

9. Voelker, G.S., Myers, P.G., Walter, M., and Sutherland, B.R., Generation of oceanic internal gravity waves by a cyclonic surface stress disturbance, Dynamics Atm. Oceans, 2019, vol. 86, pp. 116-133.

10. Haney, S. and Young, W.R., Radiation of internal waves from groups of surface gravity waves, J. Fluid Mech., 2017, vol. 829, pp. 280-303.

11. Wang, J., Wang, S., Chen, X., Wang, W., and Xu Y., Three-dimensional evolution of internal waves rejected from a submarine seamount, Phys. Fluids, 2017, vol. 29, p. 106601.

12. Belyaev, M.Yu., Decinov, L.V., Krikalev, S.K., Kumakshev, S.A., Sekerzh-Zen'kovich, S.Ya., Identification of a system of oceanic waves from space photos, Izv. Ross. Akad. Nauk. Teoriya i Systemy Upravleniya, 2009, no. 1, pp. $117-127$.

13. Morozov, E.G., Tarakanov, R.Yu., Frey, D.I., Demidova, T.A., and Makarenko, N.I., Bottom water flows in the tropical fractures of the Northern Mid-Atlantic Ridge, J. Oceanography, 2018, vol. 74, no. 2, pp. 147-167.

14. Khimchenko, E.E., Frey, D.I., and Morozov, E.G., Tidal internal waves in the Bransfield Strait, Antarctica, Russ. J. Earth. Science, 2020, vol. 20, ES2006.

15. Svirkunov, P.N. and Kalashnik, M.V., Phase patterns of dispersive waves from moving local sources, Usp. Fiz. Nauk, 2014, vol. 184, no. 1, pp. 89-100.

16. Bulatov, V.V., Vladimirov, Yu.V., and Vladimirov, I.Yu., Far fields of internal gravity waves from a source moving in the ocean with an arbitrary buoyancy frequency distribution, Russ. J. Earth Sciences, 2019, vol. 19(5), p. ES5003.

17. Bulatov, V., and Vladimirov, Yu., Generation of internal gravity waves far from moving non-local source, Symmetry, 2020, vol. 12(11), p. 1899.

18. Bulatov, V.V. and Vladimirov, Yu.V., Far fields of internal gravity waves from nonlocal perturbation sources, Protsessy v Geosredakh, 2020, no. 3(25), pp. 772-779.

19. Watson, G.N., A Treatise on the Theory of Bessel Functions (Reprint of the 2nd ed.), Cambridge: Cambridge University Press, 1995.

20. Froman, N. and Froman, P., Physical Problems Solved by the Phase-Integral Method, Cambridge: Cambridge University Press, 2002.

21. Grikurov, V.E., Phenomenon of overlapping near-acoustic zones in the near-surface waveguide and related generalization of the ray method, Izv. VUZov, Radiofizika, 1980, vol. 23, no. 9, pp. 1038-1045.

Translated by E.A. Pushkar 